\title{
ERGODIC THEOREMS FOR SEMIGROUPS OF OPERATORS
}

\author{
W. M. RUESS AND W. H. SUMMERS \\ (Communicated by Palle E. T. Jorgensen)
}

\begin{abstract}
We establish ergodic theorems for Eberlein-weakly almost periodic motions and almost-orbits of semigroups of operators. The results apply to solutions of a class of (generally, nonlinear) evolution equations.
\end{abstract}

\section{INTRODUCTION}

For a contraction semigroup $(S(t))_{t \geq 0}$ on a closed subset $C$ of a real Banach space $X$, Gutman and Pazy [21, Theorem 1] showed that whenever a motion $u=S(\cdot) x$ of $(S(t))_{t>0}$ through some $x \in C$ has (norm-) relatively compact range, then the $\Omega$-limit set $\omega(x)$ of $S(\cdot) x$ is a compact commutative group and, given any (real) Banach space $Y$ and function $f: C \rightarrow Y$ which is uniformly continuous on bounded subsets of $C$,

$$
\|\|-\lim _{T \rightarrow \infty} \frac{1}{T} \int_{0}^{T} f(S(t) x) d t=\int_{\omega(x)} f d \mu,
$$

where $\mu$ denotes normalized Haar measure on $\omega(x)$. Our basic objective is to relax the compactness requirement on the range of $u$ and extend this ergodic theorem to the context of motions or almost-orbits that only have weakly relatively compact range. Though at the expense of some restrictions on the state space $X$, this allows for a wider range of applications to (infinite-dimensional) linear and nonlinear initial value problems where norm compactness assumptions would be unduly restrictive.

The key to our development is the notion of a weakly almost periodic function in the sense introduced (in the scalar case) by Eberlein [13]. Under suitable conditions, solutions to the abstract Cauchy problem naturally fall into this category $([31,32,34,35]$ and $\S 4)$.

Received by the editors July $26,1990$.

1980 Mathematics Subject Classification (1985 Revision). Primary 47A35, 47D05, 47H20.

Key words and phrases. Semigroups of operators, mean ergodic theorem, weak almost periodicity.

This work was supported in part by the Deutsche Forschungsgemeinschaft, the National Science Foundation under Grant INT-8822565, and the Universität Essen. 


\section{Preliminaries}

Throughout the paper, $X$ denotes a (real or complex) Banach space, and $(S(t))_{t \geq 0}$ a strongly continuous semigroup of operators on a weakly closed subset $C$ of $X$.

For a function $u: \mathbb{R}^{+} \rightarrow X, \gamma(u)=\{u(t): t \geq 0\}$ denotes its range, $\omega(u)=$ $\left\{y \in X:\|\|-\lim u\left(t_{n}\right)=y\right.$ for some sequence $\left.0<t_{n} \rightarrow \infty\right\}$ its $\Omega$-limit set, and $\omega_{w}(u)=\left\{y \in X: w-\lim u\left(t_{n}\right)=y\right.$ for some sequence $\left.0<t_{n} \rightarrow \infty\right\}$ its weak $\Omega$-limit set. In case $u=S(\cdot) x$ for some $x \in C$, these sets, as usual, are denoted by $\gamma(x), \omega(x)$, and $\omega_{w}(x)$, respectively. For a subset $D$ of $X$, co $D$ denotes its convex hull, and (\|\|)- $\operatorname{cl} D$ (respectively, w-cl $D$ ) its norm(respectively, weak-) closure. $B_{X^{*}}$ denotes the closed unit ball of the dual $X^{*}$ of $X . X_{w}$ denotes $X$ with the weak topology.

For $J \in\left\{\mathbb{R}, \mathbb{R}^{+}\right\}, C_{b}(J, X)$ denotes the space of bounded continuous functions $f: J \rightarrow X$, equipped with the sup-norm. For $f \in C_{b}(J, X)$ and $t \in J$, $f_{t}(s)=f(t+s), s \in J$, and $H(f)=\left\{f_{t}: t \in J\right\}$ is the set of all translates of $f$. Recall that $f \in C_{b}(\mathbb{R}, X)$ is almost periodic [6] if $H(f)$ is relatively compact in $C_{b}(\mathbb{R}, X)$, and that a function $f: \mathbb{R} \rightarrow X$ is said to be weakly almost periodic (cf. [1]) if $x^{*} f$ is almost periodic for all $x^{*} \in X^{*}$. The space of all almost periodic functions is denoted by $\operatorname{AP}(\mathbb{R}, X)$.

1.1. Definition. A function $f \in C_{b}\left(\mathbb{R}^{+}, X\right)$ is said to be (a) asymptotically almost periodic (a.a.p.) if $H(f)$ is relatively compact in $C_{b}\left(\mathbb{R}^{+}, X\right)$ (Fréchet [15-18], and [14]), and (b) Eberlein-weakly almost periodic (E-w.a.p.) if $H(f)$ is weakly relatively compact in $C_{b}\left(\mathbb{R}^{+}, X\right)$ (Eberlein [13], and [24, 33]).

The space of all E-w.a.p. functions (endowed with the sup-norm) is denoted by $W\left(\mathbb{R}^{+}, X\right)$, while $W_{0}\left(\mathbb{R}^{+}, X\right)$ is the (closed linear) subspace of all $\phi \in$ $W\left(\mathbb{R}^{+}, X\right)$ for which the zero function belongs to the weak closure of $H(\phi)$.

In the context of the Gutman-Pazy ergodic theorem [21, Theorem 1] cited in the introduction, Theorem 2.2 of [30] shows that requiring the range of a motion $S(\cdot) x: \mathbb{R}^{+} \rightarrow X$ to be norm relatively compact implies that $S(\cdot) x$ is actually asymptotically almost periodic. After establishing the ergodic properties of general E-w.a.p. functions in $\S 2$, we devote $\S 3$ to extensions of Theorem 1 of [21] to motions with weak almost periodicity properties. In particular, we show (Theorem 3.1) that the corresponding conclusions remain valid for E-w.a.p. motions when (norm) uniform continuity of $f$ is replaced by weak uniform continuity. In $\S 4$, we discuss consequences of the results for the asymptotic behavior of solutions to the abstract Cauchy problem.

\section{ERGODIC THEOREMS FOR EBERLEIN-WEAKLY ALMOST PERIODIC FUNCTIONS}

We establish the ergodic properties of general E-w.a.p. functions. Of basic importance is the fact that these functions are uniformly continuous. In the scalar case, this was shown by Eberlein [13, Theorem 13.1], and for E-w.a.p. functions with relatively compact range in [19, Theorem 1.16].

\subsection{Proposition. If $f \in W\left(\mathbb{R}^{+}, X\right)$, then $f$ is uniformly continuous.}

Proof. According to [24, Theorem 3; 33, Theorem 2.1], a function $f \in$ $C_{b}\left(\mathbb{R}^{+}, X\right)$ belongs to $W\left(\mathbb{R}^{+}, X\right)$ if and only if, given sequences $\left(t_{m}, x_{m}^{*}\right)_{m}$ 
in $\mathbb{R}^{+} \times B_{X^{*}}$ and $\left(\omega_{n}\right)_{n}$ in $\mathbb{R}^{+}$,

$$
\lim _{n} \lim _{m}\left(f\left(t_{m}+\omega_{n}\right), x_{m}^{*}\right)=\lim _{m} \lim _{n}\left(f\left(t_{m}+\omega_{n}\right), x_{m}^{*}\right)
$$

whenever both iterated limits exist. Thus it is obvious that $f \in W\left(\mathbb{R}^{+}, X\right)$ if and only if $B_{X^{*}} \circ H(f)$ is weakly relatively compact in $C_{b}\left(\mathbb{R}^{+}\right)$. Using this criterion, we reduce the proof of Proposition 2.1 to Eberlein's arguments in the scalar case. Suppose that $f \in W\left(\mathbb{R}^{+}, X\right)$ is not uniformly continuous. Then there exist $\varepsilon>0$ and sequences $\left(s_{n}\right)_{n},\left(t_{n}\right)_{n}$ in $\mathbb{R}^{+}, 0<t_{n}-s_{n}<1 / n$, and $\left(x_{n}^{*}\right)_{n}$ in $B_{X^{*}}$ such that $\left(f\left(t_{n}\right)-f\left(s_{n}\right), x_{n}^{*}\right)=\left\|f\left(t_{n}\right)-f\left(s_{n}\right)\right\| \geq \varepsilon$ for all $n \in \mathbb{N}$. According to the above criterion, we can assume that the sequence $\left(g_{n}\right)_{n}$ with $g_{n}:=x_{n}^{*} f_{t_{n}}-x_{n}^{*} f_{s_{n}}, n \in \mathbb{N}$, converges weakly in $C_{b}\left(\mathbb{R}^{+}\right)$to some $g \in C_{b}\left(\mathbb{R}^{+}\right)$. Then, since $g(0) \geq \varepsilon$, there exists $\eta>0$ such that $\int_{0}^{\eta} g(t) d t \neq 0$. Setting $T_{\eta}(h)=\int_{0}^{\eta} h(t) d t$ for $h \in C_{b}\left(\mathbb{R}^{+}\right)$, the scalar valued map $T_{\eta}$ is an element of $C_{b}\left(\mathbb{R}^{+}\right)^{*}$, and thus we conclude that

$$
\left|T_{\eta}(g)\right|=\lim _{n}\left|T_{\eta}\left(g_{n}\right)\right|=\lim _{n}\left|\int_{s_{n}+\eta}^{t_{n}+\eta} x_{n}^{*} f(t) d t-\int_{s_{n}}^{t_{n}} x_{n}^{*} f(t) d t\right|=0 .
$$

This contradiction completes the proof.

Now we turn to the mean ergodic theorem for E-w.a.p. functions. Besides the usual "time-mean" \|\|$-\lim _{T \rightarrow \infty} \frac{1}{T} \int_{0}^{T} f(t) d t$, we also take into account means with respect to more general kernel functions (compare $[7 ; 23, \S 9.3 .4 ; 27 ; 29]$ ).

A function $Q: \mathbb{R}^{+} \times \mathbb{R}^{+} \rightarrow \mathbb{R}$ is called strongly regular if

(1) $\left\{Q(s, \cdot): s \in \mathbb{R}^{+}\right\}$is a bounded subset of $L^{1}\left(\mathbb{R}^{+}\right)$;

(2) $\lim _{s \rightarrow \infty} \int_{0}^{\infty} Q(s, t) d t=1$;

(3) $\lim _{s \rightarrow \infty} \int_{0}^{T}|Q(s, t)| d t=0$ for all $T>0$; and

(4) $\lim _{s \rightarrow \infty} \int_{0}^{\infty}|Q(s, t+h)-Q(s, t)| d t=0$ for all $h \in \mathbb{R}^{+}$.

2.2. Theorem. If $f \in W\left(\mathbb{R}^{+}, X\right)$, then there exists $z \in X$ such that \|\|$-$ $\lim _{s \rightarrow \infty} \int_{0}^{\infty} Q(s, t) f(t+h) d t=z$ uniformly over $h \in \mathbb{R}^{+}$for every strongly regular kernel $Q: \mathbb{R}^{+} \times \mathbb{R}^{+} \rightarrow \mathbb{R}$. Moreover, the function $z(t)=z$ is contained in clco $H(f)$, and is the only constant function in clco $H(f)$.

2.3. Proposition. If $\phi \in W_{0}\left(\mathbb{R}^{+}, X\right)$, then

$$
\lim _{T \rightarrow \infty} \sup \left\{\frac{1}{T} \int_{0}^{T}\left|\left(\phi(t+h), x^{*}\right)\right| d t: x^{*} \in B_{X^{*}}, h \in \mathbb{R}^{+}\right\}=0 .
$$

In the following result, we assume that $X$ is a complex Banach space. The corresponding result for a real Banach space $X$ then follows in the canonical way by complexifying both $X$ and $f \in W\left(\mathbb{R}^{+}, X\right)$.

2.4. Theorem. If $f \in C_{b}\left(\mathbb{R}^{+}, X\right)$, then $f \in W\left(\mathbb{R}^{+}, X\right)$ if and only if there exist unique functions $g \in \operatorname{AP}(\mathbb{R}, X)$ and $\phi \in W_{0}\left(\mathbb{R}^{+}, X\right)$ such that $f=$ $g_{\mid \mathbb{R}^{+}}+\phi$. Furthermore, for every $f \in W\left(\mathbb{R}^{+}, X\right)$, the Fourier coefficients

$$
a(f ; \lambda)=\|\|-\lim _{T \rightarrow \infty} \frac{1}{T} \int_{0}^{T} e^{-i \lambda t} f(t) d t
$$

exist for all $\lambda \in \mathbb{R}$ and equal the corresponding Fourier coefficients $a(g ; \lambda)$ of the almost periodic part $g$ of $f$ as specified above. 
Proofs. Proposition 2.1 serves to reduce the proof of Theorem 2.2 to the proof of the special case treated earlier [32, Theorem 2.1].

For a proof of Proposition 2.3, let $E=W\left(\mathbb{R}^{+}, X\right)$, and note that, again by Proposition 2.1 , the translation semigroup $(U(\omega))_{\omega \geq 0}$ on $E$ (where $U(\omega) g:=$ $\left.g_{\omega}\right)$ is actually a (uniformly bounded) $C_{0}$-semigroup. Next, since $\phi \in$ $W_{0}\left(\mathbb{R}^{+}, X\right)$, we conclude from [34, Theorem 2.1] that $U(\cdot) \phi \in W\left(\mathbb{R}^{+}, E\right)$. From this it easily follows that $\left|\left(U(\cdot) \phi, y^{*}\right)\right| \in W_{0}\left(\mathbb{R}^{+}\right)$, and thus

$$
\lim _{T \rightarrow \infty} \frac{1}{T} \int_{0}^{T}\left|\left(U(t) \phi, y^{*}\right)\right| d t=0,
$$

for all $y^{*} \in E^{*}$. The proof is now completed by applying [22, Theorem 2.2].

Once again considering the translation semigroup $(U(\omega))_{\omega \geq 0}$ on $E=$ $W\left(\mathbb{R}^{+}, X\right)$ and, for $f \in E$, the corresponding motion $U(\cdot) f$, the DeLeeuwGlicksberg $[10,11]$ theory yields the first part of Theorem 2.4 , while the second part is just a combination of Theorem 2.2 and Proposition 2.3.

Remark. Theorem 2.4 clarifies the extent to which the classical harmonic analysis of almost periodic functions carries over to E-w.a.p. functions. (For the scalar case, compare the problem raised at the beginning of $\S 15$ in Eberlein's classical paper [13].) As in the case for almost periodic functions, the Fourier coefficients $a(f ; \lambda)$ of an E-w.a.p. function $f$ exist and are nonzero for at most countably many $\lambda \in \mathbb{R}$, and they completely determine the almost periodic part of $f$ (via classical Bochner-Féjer approximation polynomials). However, $f$ itself is only determined up to an additive factor $\phi \in W_{0}\left(\mathbb{R}^{+}, X\right)$. (For a version of the second part of Theorem 2.4 for functions with relatively compact range, see [19, Theorem 5.1].)

\section{ERGODIC THEOREMS FOR ALMOST-ORBITS OF OPERATOR SEMIGROUPS}

A continuous function $u: \mathbb{R}^{+} \rightarrow C$ is said to be an almost-orbit of $(S(t))_{t \geq 0}$ $\left[25\right.$, p. 351] if $\lim _{t \rightarrow \infty} \sup _{h \in \mathbb{R}^{+}}\|u(t+h)-S(h) u(t)\|=0$. Under suitable conditions, solutions to both the linear and nonlinear Cauchy problem are almostorbits of the semigroup associated with the problem (see $\S 4)$.

Throughout this section, we consider almost-orbits $u$ of $(S(t))_{t \geq 0}$ with the following additional property:

$$
\begin{aligned}
& S(h)_{\mid \text {w-cl } \gamma(u)}: \text { w-cl } \gamma(u) \rightarrow X \text { is weak-to-weak continuous for all } \\
& h \in \mathbb{R}^{+} .
\end{aligned}
$$

This is equivalent to assuming that, for a sequence $0<t_{n} \rightarrow \infty$, if $\left(u\left(t_{n}\right)\right)_{n}$ is weakly convergent to $c \in C$, then $w$ - $\lim S(h) u\left(t_{n}\right)=S(h) c$ for all $h \in \mathbb{R}^{+}$ [34, Remark 2.5]. Condition (3.0) is fulfilled in the following cases:

(i) $\gamma(u)$ is relatively compact.

(ii) $(S(t))_{t \geq 0}$ consists of linear operators.

(iii) $X$ is uniformly convex, $(S(t))_{t \geq 0}$ is a contraction semigroup on a closed convex subset $C$ of $X$, and $u$ is bounded and asymptotically isometric [8]; i.e., $\lim _{t \rightarrow \infty}\|u(t+h)-u(t)\|=\rho(h)$ exists uniformly over $h \in \mathbb{R}^{+}$(see [32, Lemma 1.7]).

In this section we write $(D, w)$ to denote a subset $D$ of $X$ endowed with the weak topology induced by $X$. Also, we call a function $f: \mathbb{R}^{+} \rightarrow X$ almost periodic if it is the restriction to $\mathbb{R}^{+}$of a function $g \in \operatorname{AP}(\mathbb{R}, X)$. 
3.1. Theorem. If $u$ is an E-w.a.p. almost-orbit satisfying (3.0), then the following propositions hold:

(a) There exist unique elements $y \in \omega_{w}(u)$ and $\phi \in W_{0}\left(\mathbb{R}^{+}, X\right)$ such that

(i) $u=S(\cdot) y+\phi$, and

(ii) $S(\cdot) y$ is almost periodic.

(b) The $\Omega$-limit set $\omega(y)$ of $S(\cdot) y$ (with the norm topology) carries the structure of a compact commutative group. Moreover, taking $\mu$ to denote normalized Haar measure on $\omega(y)$ and $K=(\mathrm{w}-\mathrm{cl} \gamma(u), w)$, if $Y$ is any Banach space, then

(i) $w-\lim _{T \rightarrow \infty} \frac{1}{T} \int_{0}^{T} f(u(t+h)) d t=\int_{\omega(y)} f d \mu$ for all $f \in C\left(K, Y_{w}\right)$, and

(ii) \|\|$-\lim _{T \rightarrow \infty} \frac{1}{T} \int_{0}^{T} f(u(t+h)) d t=\int_{\omega(y)} f d \mu$ for all $f \in C(K, Y)$, and all bounded linear operators $f: X \rightarrow Y$,

where both limits exist uniformly over $h \in \mathbb{R}^{+}$.

Proof. Proposition (a) is taken from [34, Theorem 2.4]. As for proposition (b), it is enough to establish (b)(ii), because (b)(i) then follows directly from the fact that any $f \in C\left(K, Y_{w}\right)$ can be approximated uniformly (over $K$ )-weakly (in $Y$ ) by functions of the form $\sum_{i=1}^{n} \phi_{i}(\cdot) y_{i}$ with $\phi_{i} \in C(K)$ and $y_{i} \in Y$ for $i \in\{1, \ldots, n\}, n \in \mathbb{N}$. For the proof of (b)(ii), we first observe that the $\Omega$-limit set $\omega(y)$ of the almost periodic motion $S(\cdot) y$ from (a) carries the structure of a compact commutative group (combine [30, Theorem 2.2; 12, Theorem 3; and 26, Part 2, Chapter V, Theorem 8.16]). Next we need the following technical result for arbitrary Banach spaces $X$ and $Y$.

3.2. Lemma. If $g \in W\left(\mathbb{R}^{+}, X\right)$ and $K=(\mathrm{w}-\mathrm{cl} \gamma(g), w)$, then $f \circ g \in$ $W\left(\mathbb{R}^{+}, Y\right)$ for all $f \in C(K, Y)$.

This can be shown by using the iterated-double-limits criterion for E-w.a.p. functions [24, Theorem 3; 33, Theorem 2.1].

Returning to the proof of Theorem 3.1(b)(ii), if $f \in C(K, Y)$, then combining Theorem 2.2 from $\S 2$ with Lemma 3.2, both

$$
\|\|-\lim _{T \rightarrow \infty} \frac{1}{T} \int_{0}^{T} f(u(t+h)) d t \text { and }\|\|-\lim _{T \rightarrow \infty} \frac{1}{T} \int_{0}^{T} f(S(t+h) y) d t
$$

exist uniformly over $h \in \mathbb{R}^{+}$. Invoking [26, Part 2, Chapter VI.6, Theorem 6.05 and Chapter VI.9, Theorem 9.34], we conclude that

$$
\|\|-\lim _{T \rightarrow \infty} \frac{1}{T} \int_{0}^{T} f(S(t+h) y) d t=\int_{\omega(y)} f d \mu \quad \text { uniformly over } h \in \mathbb{R}^{+} .
$$

Now let $z=\|\|-\lim _{T \rightarrow \infty} \frac{1}{T} \int_{0}^{T}(f(u(t+h))-f(S(t+h) y)) d t$ uniformly over $h \in \mathbb{R}^{+}$; it remains to show that $z=0$. For this, given $\varepsilon>0$, since $f \in$ $C(K, Y)$, there exists a weak zero neighborhood $U$ in $X$ such that

$$
c_{1}, c_{2} \in K \text { and } c_{1}-c_{2} \in U \text { imply }\left\|f\left(c_{1}\right)-f\left(c_{2}\right)\right\|<\varepsilon .
$$

Also, there exists $T>0$ such that

$$
\left\|\frac{1}{T} \int_{0}^{T}(f(u(t+h))-f(S(t+h) y)) d t-z\right\|<\varepsilon \text { for all } h \in \mathbb{R}^{+} .
$$


Finally, consider the critical part $\phi \in W_{0}\left(\mathbb{R}^{+}, X\right)$ of the decomposition of $u$ specified in (a)(i). Since there exists a sequence $\left(\phi_{\omega_{n}}\right)_{n}$ of translates of $\phi$ that converges weakly (in $W\left(\mathbb{R}^{+}, X\right)$ ) to the zero function, and $\phi$ is uniformly continuous (Proposition 2.1), $\left(\phi_{\omega_{n}}\right)_{n}$ converges to the zero function weakly in $X$ and uniformly on compact subsets of $\mathbb{R}^{+}$. Thus, there exists $n \in \mathbb{N}$ such that

$$
\phi_{\omega_{n}}(t) \in U \text { for all } t \in[0, T] .
$$

Using the decomposition of (a)(i) for $u$, this implies that

$$
u\left(t+\omega_{n}\right)-S\left(t+\omega_{n}\right) y \in U \text { for all } t \in[0, T] .
$$

Combining (1), (2), and (4), we conclude that $\|z\|<2 \varepsilon$. Consequently, $z=0$, and the proof of Theorem 3.1 is complete.

Without going into detail, we present an analog of Theorem 3.1 for a concept of weak almost periodicity for functions on $\mathbb{R}^{+}$that corresponds to weak almost periodicity for functions defined on all of $\mathbb{R}$; a function $f: \mathbb{R}^{+} \rightarrow X$ is said to be weakly asymptotically almost periodic [33] if $x^{*} f$ is asymptotically almost periodic for each $x^{*} \in X^{*}$.

3.3. Theorem. If $u$ is a weakly asymptotically almost periodic almost-orbit with weakly relatively compact range satisfying (3.0), then we have the following:

(a) There exist unique elements $y \in \omega_{w}(u)$ and $\phi \in C_{0}\left(\mathbb{R}^{+}, X_{w}\right)$ such that

(i) $u=S(\cdot) y+\phi$ and

(ii) $S(\cdot) y$ is weakly almost periodic with weakly relatively compact range.

(b) The weak $\Omega$-limit set $\left(\omega_{w}(u), w\right)$ of $u$ with the weak topology carries the structure of a compact commutative group. If $\mu$ denotes normalized Haar measure on $\left(\omega_{w}(u), w\right), K=(\mathrm{w}-\mathrm{cl} \gamma(u), w)$, and $Y$ is any Banach space, then

(i) $w-\lim _{T \rightarrow \infty} \frac{1}{T} \int_{0}^{T} f(u(t+h)) d t=\int_{\omega_{w}(u)} f d \mu$ for all $f \in C\left(K, Y_{w}\right)$, and

(ii) \|\|$-\lim _{T \rightarrow \infty} \frac{1}{T} \int_{0}^{T} f(u(t+h)) d t=\int_{\omega_{w}(u)} f d \mu \quad$ for all $f \in C(K, Y)$, where both limits exist uniformly over $h \in \mathbb{R}^{+}$.

Proposition (a) is taken from [36, Theorem 2.2]. Concerning the rest of the proof, except for minor changes due to the different concept of weak almost periodicity, this follows along similar lines as that given for Theorem 3.1, and therefore we omit the argument.

The following example shows that the ergodic assertions (b)(i) and (b)(ii) of both Theorems 3.1 and 3.3 can fail to hold if $f$ is only norm continuous from $K=$ w-cl $\gamma(u)$ into either $Y_{w}$ or $Y$.

3.4. Example. Consider the $C_{0}$-semigroup $(S(t))_{t \geq 0}$ of linear operators on $X=L^{2}\left(\mathbb{R}^{+}\right)$defined by

$$
S(t) g(\tau)= \begin{cases}g(\tau-t), & \tau \geq t \\ 0, & 0 \leq \tau<t\end{cases}
$$


for $g \in X$ and $t, \tau \in \mathbb{R}^{+}$(cf. [3, pp. 202-203]). According to [34, Theorem 2.1], if $u=S(\cdot) g$ is the motion through any $g \in X$, then $u \in W\left(\mathbb{R}^{+}, X\right)$. Since $u \in C_{0}\left(\mathbb{R}^{+}, X_{w}\right)$, moreover, $u$ is also weakly asymptotically almost periodic, and $K=\mathrm{w}-\mathrm{cl} \gamma(u)=\gamma(g) \cup\{0\}$. Now let $g \in X$ be the element defined by $g(\tau)=\exp (-\tau), \tau \in \mathbb{R}^{+}$. In this case, since there exists a unique $t \in \mathbb{R}^{+}$ such that $y=S(t) g$ for each $y \in \gamma(g)$, we put

$$
f(y)= \begin{cases}\|\sin \log (1+t) S(t) g\|, & t \in \mathbb{R}^{+} \text {and } y=S(t) g, \\ 0 & y=0 .\end{cases}
$$

Then $f: K \rightarrow \mathbb{R}$ is uniformly continuous when $K$ has the relative norm topology from $X$, but $\lim _{T \rightarrow \infty} \frac{1}{T} \int_{0}^{T} f(S(t) g) d t$ does not exist.

3.5. Remarks. 1. If the almost-orbit $u: \mathbb{R}^{+} \rightarrow C$ is both an element of $W\left(\mathbb{R}^{+}, X\right)$ and weakly asymptotically almost periodic, then $\omega_{w}(u)=\omega(y)$, which implies that $\omega_{w}(u)$ itself is a norm-compact commutative group. In this case, therefore, proposition (b) of both Theorems 3.1 and 3.3 holds for $\omega_{w}(u)=\omega(y)$, where $\omega_{w}(u)$ is endowed with the norm topology.

2. If $u \in W\left(\mathbb{R}^{+}, X\right)$ has (norm-) relatively compact range, then Theorem 3.1 holds with $K=\|\|-\operatorname{cl} \gamma(u)$ endowed with the norm topology.

3. According to the preceding remarks, Theorems 3.1 and 3.3 both include the Gutman-Pazy ergodic result [21, Theorem 1] as a special case.

\section{Applications to solutions of the Cauchy problem}

The foregoing results can be applied to solutions of both the homogeneous and, for a given function $g \in L^{1}\left(\mathbb{R}^{+}, X\right)$, the inhomogeneous Cauchy problem

$$
\left\{\begin{array}{l}
\dot{u}(t) \in A u(t), \quad t \in \mathbb{R}^{+} \\
u(0)=u_{0} \in \operatorname{cl} D(A)
\end{array}\right.
$$

and

$$
\left\{\begin{array}{l}
\dot{u}(t) \in A u(t)+g(t), \quad t \in \mathbb{R}^{+} \\
u(0)=u_{0} \in \operatorname{cl} D(A)
\end{array}\right.
$$

in both (a) the linear case and (b) the nonlinear case.

4.1. Assumptions. (a) For the linear case, $X$ is an (arbitrary) Banach space and $A: D(A) \subset X \rightarrow X$ is a closed linear operator which is the infinitesimal generator of a uniformly bounded $C_{0}$-semigroup $(S(t))_{t \geq 0}$ of continuous linear operators on $X$.

(b) For the nonlinear case, $X$ is a uniformly convex Banach space, $A$ is a $m$-dissipative (generally multivalued) operator in $X$, and $(S(t))_{t \geq 0}$ is the contraction semigroup on $C=\operatorname{cl} D(A)$ generated by $A$ (in the sense of CrandallLiggett [9]).

Then (cf. $[4,20,28])$ strong solutions to $\left(\mathrm{CP}_{\mathrm{h}}\right)$ are motions of the associated semigroup $(S(t))_{t \geq 0}$, and the mild solution $u(t)=S(t) u_{0}+\int_{0}^{t} S(t-s) g(s) d s$ (linear case), respectively, the integral solution in the sense of Bénilan [5] (nonlinear case) to (CP) is an almost-orbit of $(S(t))_{t \geq 0}$. 
4.2. Theorem. Under the assumptions 4.1 , let $u: \mathbb{R}^{+} \rightarrow X$ be a strong solution to $\left(C P_{h}\right)$ or the mild (linear case), respectively, integral (nonlinear case) solution to $(C P)$. Also assume that

(a) $u$ has weakly relatively compact range in the linear case, or

(b) $u$ is bounded and asymptotically isometric (i.e., $\lim _{t \rightarrow \infty}\|u(t+h)-u(t)\|=$ $\rho(h)$ exists uniformly over $\left.h \in \mathbb{R}^{+}\right)$in the nonlinear case.

Then the conclusions of Theorem 3.1 hold for $u$. Moreover, if the set of operators $\left\{S(h)_{\mid \gamma(u)}: h \in \mathbb{R}^{+}\right\}$is weak-to-weak uniformly equicontinuous on $\gamma(u)$, then these conclusions hold when $\omega(y)$ is replaced by $\omega_{w}(u)$.

This follows by combining Theorem 3.1 and Remark 3.5.1 with our previous results [34, Theorem 3.1; 31, Théorème $1 ; 32$, Theorem 1.4 and Lemma 1.7; 36, Theorem 4.1 and Proposition 4.1.2].

4.3. Remarks on Theorem 4.2. 1. Theorem 4.2 shows in particular that such a process $u \in W\left(\mathbb{R}^{+}, X\right)$ as well as compositions with certain "observables" $f$ are ergodic in the sense that the limit of the time-means along (and starting from any point of the trajectory $\gamma(u)$ exists and is equal to the space mean of $f$ over the $\Omega$-limit set $\omega(y)$ of the almost periodic part $S(\cdot) y$ of $u$.

2 . The condition that $u$ be asymptotically isometric in the nonlinear case is automatically fulfilled in the following cases:

(a) For an individual almost-orbit, if $\omega(u) \neq \varnothing$ [25, Proposition 5.5].

(b) For every almost-orbit $u$ of $(S(t))_{t \geq 0}$, if $X$ is a Hilbert space, $0 \in C=$ $\operatorname{cl} D(A)$, and there exists $L>0$ such that

$$
\|S(t) x+S(t) y\|^{2} \leq\|x+y\|^{2}+L\left\{\|x\|^{2}-\|S(t) x\|^{2}+\|y\|^{2}-\|S(t) y\|^{2}\right\}
$$

for all $t \in \mathbb{R}^{+}, x, y \in C$ [25, Corollary 6.2]. In particular, this is true if $X$ is a Hilbert space, $C=-C$, and each $S(t), t \in \mathbb{R}^{+}$, is odd [2; 23, §9.3].

3 . In the linear case, the extra condition on $u$ concerning the set of operators $\left\{S(h)_{\mid \gamma(u)}: h \in \mathbb{R}^{+}\right\}$is automatically satisfied if all dual motions $S(\cdot)^{*} x^{*}: \mathbb{R}^{+} \rightarrow$ $X^{*}$ have (norm-) relatively compact range [36, Corollary 4.3.2 and Theorem 4.1.1].

4. We note the following specific examples of "observables" $f \in C(K, Y)$ :

4.1. Finite-dimensional approximations: (a) If $X=Y$ is a Banach space with a projection scheme $\left(X_{n}\right)_{n}$ of finite-dimensional subspaces $X_{n}$, let $f=$ $P_{n}: X \rightarrow X_{n}$ be the projection onto $X_{n}$.

(b) If $X=Y=H$ is a real Hilbert space and $C$ is a closed convex boundedly compact (i.e., bounded subsets of $C$ are relatively compact) subset of $H$, let $f: H \rightarrow C$ be the best approximation projection onto $C$.

4.2. Compact embeddings: If $j: X \rightarrow Z$ is a compact linear embedding and $g: j(X) \rightarrow Y$ is continuous, then $f=g \circ j_{\mid K} \in C(K, Y)$. In applications to partial differential equations, for example, where $u(t) \in D(A) \subset H_{0}^{1}(\Omega)$, $\Omega$ bounded and open in $\mathbb{R}^{n}$, this yields (under the assumptions of Theorem 4.2) the existence of $\lim _{T \rightarrow \infty} \frac{1}{T} \int_{0}^{T}\|u(t)\|_{2}^{s} d t, s>0$, where $\|\cdot\|_{2}$ denotes the $L^{2}(\Omega)$-norm. 


\section{REFERENCES}

1. L. Amerio and G. Prouse, Almost-periodic functions and functional equations, Van Nostrand, New York, 1971.

2. J. B. Baillon, Quelques propriétés de convergence asymptotique pour les semi-groupes de contractions impaires, C. R. Acad. Sci. Paris Sér. A-B 283 (1976), A75-A78.

3. A. V. Balakrishnan, Applied functional analysis, Appl. Math., vol. 3, Springer-Verlag, New York, 1981.

4. V. Barbu, Nonlinear semigroups and differential equations in Banach spaces, Noordhoff, Leyden, 1976.

5. Ph. Bénilan, Solutions intégrales d'équations d'évolution dans un espace de Banach, $\mathrm{C} . \mathrm{R}$. Acad. Sci. Paris Sér. A-B 274 (1972), A47-A50.

6. S. Bochner, Abstrakte fastperiodische Funktionen, Acta Math. 61 (1933), 149-184.

7. H. Brézis, Asymptotic behavior of some evolution systems, Nonlinear evolution equations, (M. G. Crandall, ed.), Academic Press, New York, 1978, pp. 141-154.

8. R. E. Bruck, On the almost-convergence of iterates of a nonexpansive mapping in Hilbert space and the structure of the weak w-limit set, Israel J. Math. 29 (1978), 1-16.

9. M. G. Crandall and T. M. Liggett, Generation of semigroups of nonlinear transformations on general Banach spaces, Amer. J. Math. 93 (1971), 265-298.

10. K. DeLeeuw and I. Glicksberg, Applications of almost periodic compactifications, Acta Math. 105 (1961), 63-97.

11. __ Almost periodic functions on semigroups, Acta Math. 105 (1961), 99-140.

12. G. Della Riccia, Equicontinuous semi-flows (one-parameter semigroups) on locally compact or complete metric spaces, Math. Systems Theory 4 (1970), 29-34.

13. W. F. Eberlein, Abstract ergodic theorems and weak almost periodic functions, Trans. Amer. Math. Soc. 67 (1949), 217-240.

14. K. Fan, Les fonctions asymptotiquement presque-périodiques d'une variable entière et leur application à l'étude de l'itération des transformations continues, Math. Z. 48 (1943), 685711.

15. M. Fréchet, Les fonctions asymptotiquement presque-périodiques continues, C. R. Acad. Sci. Paris 213 (1941), 520-522.

16. __ Les fonctions asymptotiquement presque-périodiques, Revue Sci. 79 (1941), 341-354.

17. __ Une application des fonctions asymptotiquement presque-périodiques à l'étude des familles de transformations ponctuelles et au problème ergodique, Revue Sci. 79 (1941), 407-417.

18. Les transformations asymptotiquement presque-périodiques discontinues et le lemme ergodique, Proc. Royal Soc. Edinburgh 63 (1949-50), 61-68.

19. S. Goldberg and P. Irwin, Weakly almost periodic vector-valued functions, Dissertationes Math. 157 (1979), 46pp.

20. J. A. Goldstein, Semigroups of operators and applications, Oxford Math. Monographs, Oxford Univ. Press, New York, 1985.

21. S. Gutman and A. Pazy, An ergodic theorem for semigroups of contractions, Proc. Amer. Math. Soc. 88 (1983), 254-256.

22. F. Hiai, Weakly mixing properties of semigroups of linear operators, Kodai Math. J. 1 (1978), 376-393.

23. U. Krengel, Ergodic theorems, De Gruyter Studies in Math., vol. 6, de Gruyter, Berlin, 1985.

24. P. Milnes, On vector-valued weakly almost periodic functions, J. London Math. Soc. (2) 22 (1980), 467-472.

25. I. Miyadera and K. Kobayasi, On the asymptotic behavior of almost-orbits of nonlinear contraction semigroups in Banach spaces, Nonlinear Anal. 6 (1982), 349-365.

26. V. V. Nemytskii and V. V. Stepanov, Qualitative theory of differential equations, Princeton Univ. Press, Princeton, NJ, 1960. 
27. A. Pazy, Remarks on nonlinear ergodic theory in Hilbert space, Nonlinear Anal. 3 (1979), 863-871.

28. __ Semigroups of linear operators and applications to partial differential equations, Appl. Math. Sci., vol. 44, Springer-Verlag, New York, 1983.

29. S. Reich, Almost convergence and nonlinear ergodic theorems, J. Approx. Theory 24 (1978), 269-272.

30. W. M. Ruess and W. H. Summers, Minimal sets of almost periodic motions, Math. Ann. 276 (1986), 145-158.

31. __ Presque-périodicité faible et théorème ergodique pour les semi-groupes de contractions non linéaires, C. R. Acad. Sci. Paris Sér. I Math. 305 (1987), 741-744.

32. __ Weak almost periodicity and the strong ergodic limit theorem for contraction semigroups, Israel J. Math. 64 (1988), 139-157.

33. __ Integration of asymptotically almost periodic functions and weak asymptotic almost periodicity, Dissertationes Math. 279 (1989), 38pp.

34. __, Weakly almost periodic semigroups of operators, Pacific J. Math. 143 (1990), 175-193.

35. __, Weak almost periodicity and the strong ergodic limit theorem for periodic evolution systems, J. Funct. Anal. 94 (1990), 177-195.

36. __ Weak asymptotic almost periodicity for semigroups of operators, J. Math. Anal. Appl. (to appear).

Fachbereich Mathematik, Universität Essen, D-4300 Essen 1, Federal Republic of GerMANY

Department of Mathematical Sciences, University of Arkansas, Fayetteville, ARKANSAS 72701 